\title{
Patrones biogeográficos de las aves de la península de Yucatán
}

\author{
Biogeographical patterns of the Yucatán Peninsula birds
}

\author{
Gala Cortés-Ramírez ${ }^{\bowtie}$, Alejandro Gordillo-Martínez y Adolfo G. Navarro-Sigüenza \\ Museo de Zoología, Facultad de Ciencias, Universidad Nacional Autónoma de México. Apartado postal 70-399, 04510 México, D. F., México. \\ \galamon01@gmail.com
}

\begin{abstract}
Resumen. Con el propósito de actualizar el conocimiento de la avifauna (residente, migratoria y endémica) de la Península de Yucatán, se analizaron datos de distribución puntual y distribuciones potenciales obtenidas mediante modelos de nicho ecológico a partir de la información obtenida de colecciones científicas. Para examinar la similitud entre la avifauna, se utilizó un índice de distancia fenética y se estimó el número probable de especies que se distribuye en la región. El número de registros en la base de datos quedó conformado por la presencia de 436 especies de aves (de 440 estimadas), 5 de las cuales son endémicas de México y se distribuyen en isla Cozumel y en la parte norte de la península, donde se encuentran las zonas de mayor aridez, el bosque tropical seco y 17 especies cuasiendémicas que se distribuyen en el resto de la península. Se reconocieron 4 principales agrupamientos de similitud faunística; uno al norte, otro en el área central, uno más al sur de la península y el cuarto en isla Cozumel.
\end{abstract}

Palabras clave: aves, patrones de distribución, análisis de similitud, índice Bray-Curtis, península de Yucatán.

\begin{abstract}
We analyzed geographical distribution patterns for the resident, migratory and endemic birds of the Yucatán Peninsula, using data obtained in scientific collections and predicted distributional areas obtained by ecological niche models with the purpose of updating the knowledge about the birds of the area. We examined the similarity between the bird components using a phenetic distance index, and estimated the possible number of species distributed in the peninsula. We recorded the presence of 436 species (of 440 estimated) with the information of database. Five species are endemic to Mexico and 17 quasiendemic. Mexican endemics are distributed in Cozumel Island and in the northwestern portion of the peninsula, in dryer areas with tropical dry forest. Quasiendemic species are distributed in the southeastern portion of the peninsula, mainly in areas with high humidity. Similarity analysis lead to the recognition of 4 major areas, one in the north of the peninsula, the second in the central area, the third in the south, and the fourth being Cozumel Island.
\end{abstract}

Key words: birds, distributional patterns, similarity analysis, Bray-Curtis index, Yucatán Peninsula.

\section{Introducción}

La comprensión de los patrones de distribución de las especies es crucial tanto para el conocimiento como para la conservación de la biodiversidad de México. Su importancia es aún mayor si se considera la acelerada pérdida de la biodiversidad debida principalmente a la destrucción y fragmentación del hábitat (Myers, 1993). Este deterioro ambiental pone en peligro los bienes y servicios que se obtienen del correcto funcionamiento de los ecosistemas (Gómez-Pompa, 2004). El problema se acentúa en la península de Yucatán, donde la ganadería y agricultura han desplazado gran parte de la vegetación original (Hernández-Barrios y García de Fuentes, 2002)

La península de Yucatán es una de las áreas de México donde es posible encontrar mayor riqueza de especies

Recibido: 16 agosto 2010; 07 septiembre 2011
(Escalante et al., 1998; Sánchez y Pérez-Hernández, 2005); además, es un importante centro de endemismo ornitológico (Paynter, 1955a), y sitio de paso y estancia de aves migratorias neárticas (Lynch, 1989). Por lo anterior, la avifauna de la región ofrece una excelente oportunidad para analizar los patrones de distribución de las aves y comparar los resultados con grupos previamente analizados.

El estudio de la biota de la península de Yucatán se inició desde muy temprano con la exploración biológica de México (e.g. Salvin y Godman, 1879-1904; Ridgway, 1881; Boucard, 1883; Ridgway, 1882, 1885a, b y c; Salvin, 1888-890; Cole et al., 1906; Dwight y Griscom, 1927; Van Tyne y Trautman, 1941; Goldman, 1951; Paynter, 1953, 1955a y b), la cual ha generado diversos trabajos biogeográficos concernientes a diferentes grupos de plantas y vertebrados (e.g., Barrera, 1962; Arita y Vázquez-Domínguez, 2003; Espadas-Manrique et al., 2003; Ramírez-Barahona et al., 2009). Los realizados 
en específico sobre la avifauna abarcan temas de interés para su conocimiento y conservación (véase MacKinnon, 1993 y Rodríguez-Yáñez et al., 1994). Gran parte de estos trabajos se enfoca en interacciones ecológicas de ciertas especies de aves, en especial migratorias neotropicales, con otros elementos del medio (e.g., Van Tyne y Trautman, 1945; Burskirk, 1968; Waide et al., 1980; Scott y Martin, 1984; Greenberg et al., 1993; Deppe et al., 2005). Por otra parte, se ha dedicado un gran esfuerzo a completar la lista de especies de aves presentes en la península de Yucatán y su distribución con trabajos importantes desde principios del siglo XX (e.g., Peters, 1913; Griscom, 1926; Traylor, 1941, Paynter, 1955a) hasta los últimos años (e.g., Waide et al., 1980; Madariaga y Yerena de V., 1981; Waide, 1981; Scott et al., 1985; Rogers et al., 1986; García y Correa-Sandoval, 1988, García et al., 1989; Howell, 1989; MacKinnon, 1989; López-Ornat et al., 1989; Lynch, 1989; López-Ornat y Ramo, 1992; Lynch, 1992; MacKinnon, 1992, 1993; Parkes, 1997; IbáñezHenández, 2000; Collins, 2003; MacKinnon et al., 2003; MacAndrews et al., 2006; Correa Sandoval et al., 2006; Chablé-Santos et al., 2011).

La península de Yucatán está reconocida como una provincia biogeográfica porque posee una flora y fauna particular, y por ser una unidad fisiográfica separada de las adyacentes (Morrone et al., 2002). Sin embargo, como en otros casos en México, los límites geográficos de varias provincias no siempre son claros; de hecho, si se obtiene información adicional adecuada y precisa sobre la distribución de las especies y las causas de esta distribución, puede subdividirse en zonas definidas de manera natural (Ibarra-Manríquez et al., 2002; Morrone et al., 2002).

Algunos trabajos previos incluyen el análisis de la distribución de la avifauna de la región con base en diferentes metodologías. Uno de los estudios pioneros sobre regionalización es el de Goldman y Moore (1946), quienes encontraron que en la península es posible reconocer 3 áreas de similitud faunística o distritos bióticos a partir de la distribución de plantas, mamíferos y aves, uno en la parte noroeste de la península, otro al sureste y el tercero, la isla de Cozumel.

El trabajo de Paynter (1955a) es hasta la fecha el estudio ornitológico más completo realizado en la región. En él se abordaron los datos de distribución geográfica y preferencias de hábitat de 429 especies de aves de la península, así como explicaciones sobre la composición, distribución y origen de la avifauna. Paynter propone que esta avifauna se deriva de áreas adyacentes; reconoce 3 componentes avifaunísticos en la región: el continental, el antillano (islas del Caribe) y el endémico, destacando que la península es baja en endemismos y que la distri- bución de las especies endémicas se ubica en áreas de baja precipitación. Debido a la escasez de elementos endémicos, el hecho de que el área sea geológicamente joven, así como la falta de características geográficas que pudieran funcionar como barreras, Paynter infiere que el endemismo de la península se conforma por especies de distribución relictual que alcanzaron la zona lo suficientemente temprano como para diferenciarse en especies, o bien, porque evolucionaron muy rápido.

Por otro lado, Peterson et al. (1998) analizan la avifauna residente y endémica del norte de Centroamérica mediante el método de ligamiento promedio no ponderado (UPGMA) utilizando provincias bióticas como unidades geográficas, y como datos de distribución, los registros de bases de datos e información contenida en las descripciones de AOU (1983). Los autores encuentran una diferenciación entre el norte y sur de la península. Sin embargo, la región analizada aborda una gran área que no sólo incluye la península de Yucatán.

Por todo lo anterior, en este trabajo se analiza la distribución de la riqueza y el endemismo de la avifauna de la península de Yucatán a partir de registros en ejemplares de colecciones científicas y de distribuciones potenciales obtenidas mediante modelos de nicho ecológico, con la finalidad de mejorar el conocimiento de dichos patrones en la provincia y diferenciar aquellos que pudieran asociarse con la geografía y clima del lugar. Los patrones estudiados en este análisis, contribuyen a la discusión de la composición avifaunística de la península de Yucatán, además, de que proporcionan información básica de la diversidad de aves y su distribución en la región y el país, datos que son de importancia en la planeación, elaboración y aplicación de políticas de conservación.

\section{Materiales y métodos}

Desde el punto de vista biogeográfico, la península de Yucatán comprende el territorio de los estados de Campeche, Quintana Roo y Yucatán, así como áreas de Tabasco, Chiapas, Guatemala y el norte de Belice, con lo que su

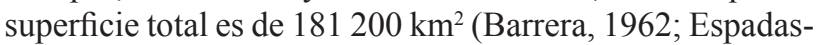
Manrique et al., 2003). Sin embargo, para propósitos de este trabajo, por la accesibilidad de la información, sólo se consideró la distribución de las aves en los 3 primeros estados, que son los que conforman el núcleo de la península. Esta área se encuentra en las coordenadas extremas $17^{\circ} 48^{\prime}-21^{\circ} 35^{\prime} \mathrm{N}, 86^{\circ} 43^{\prime}-92^{\circ} 27^{\prime} \mathrm{O}$, sin incluir las islas (Beltrán, 1958; Fig. 1).

La base de datos de la avifauna de la península de Yucatán se conformó con el programa Access (Microsoft, 2003); incluye los siguientes rubros: nombre de la especie, familia y orden; localidad, estado, longitud, latitud, fecha 


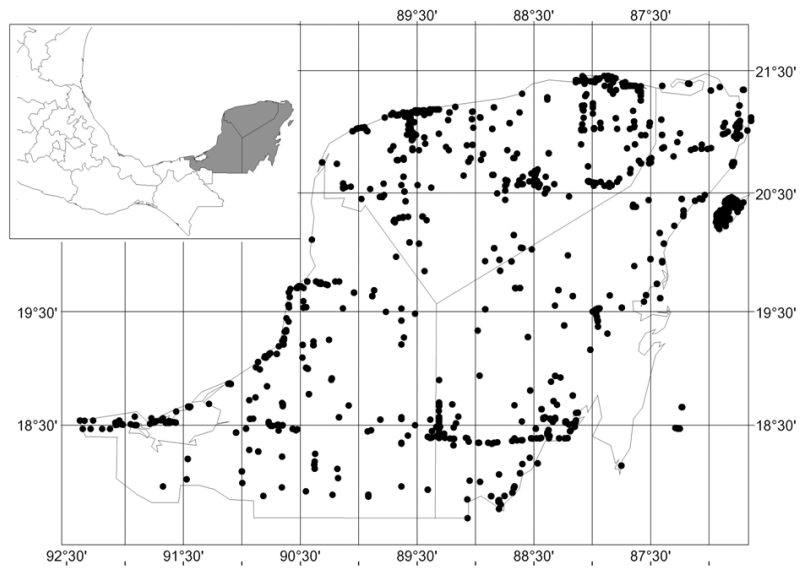

Figura 1. Ubicación geográfica de la península de Yucatán y puntos de presencia de las aves registradas.

de colecta, estacionalidad, endemismo y categoría en la NOM-059-ECOL-2001 (SEMARNAT, 2002). Estos datos se obtuvieron principalmente del "Atlas de las aves de México" (Navarro et al., 2003), que contiene registros de ejemplares de aves mexicanas tomados de diversas fuentes en el mundo, de la Colección Nacional de Aves del Instituto de Biología, UNAM y de la Colección Ornitológica del Laboratorio de Vertebrados Terrestres, IPN. Finalmente, se consultaron fuentes bibliográficas para completar lo concerniente a la distribución (Paynter, 1955a; Hardy et al., 1975; Howell, 1989; López-Ornat et al., 1989; Lynch, 1989; López-Ornat y Ramo, 1992; DeGraaf y Rappole, 1995; AOU, 1998; Kluza, 1998).

El arreglo filogenético es el propuesto por AOU (1998) y sus suplementos 42-50 (AOU, 2000; Banks et al., 20022008 y Chesser et al., 2009). La estacionalidad de cada especie se obtuvo a partir de los datos de presencia mensual de los registros y con apoyo en la bibliografía (e.g., DeGraaf y Rappole, 1995; Howell y Webb, 1995; AOU, 1998). De acuerdo con Navarro y Benítez (1993) se asignaron a las aves 5 categorías estacionales: residentes, que son las que se encuentran presentes en México todo el año; migratorias de invierno, las que pasan el invierno en México y se reproducen en Estados Unidos de América y Canadá; migratorias de verano, que sólo se encuentran en México en la etapa reproductiva pero el resto del año lo pasan en Sudamérica; transitorias, se encuentran en la zona por un corto tiempo, ya que sólo la utilizan como parte de su ruta de migración hacia otro lugar, y ocasionales $o$ accidentales, aquellas que se encuentran en la zona sólo cuando se desvían de su ruta de migración debido a condiciones atmosféricas anormales o que sus visitas al país se dan en ciclos largos. La información sobre endemismo fue establecida de acuerdo con AOU (1998) y dividida en 2 categorías: endémicas de México (EM) y cuasiendémicas (CE); estas últimas se encuentran tanto en la porción mexicana de la península como en Belice y Guatemala.

La riqueza de especies y el endemismo se calcularon en la base de datos para toda la península con el fin de estimar el número total de especies que se distribuyen en la región. A partir de los registros, se aplicó el estimador de riqueza Chao2 en el programa EstimateS 8.2 (Colwell, 2009). Este estimador permite calcular la riqueza total real de especies que puede presentarse en un área determinada basándose en la fauna ya registrada en un conjunto de muestras (Escalante et al., 2002; Jonson y Ward, 2002).

La península se dividió en cuadrantes de $0.25^{\circ} \times 25^{\circ}$ que en estas latitudes equivale aproximadamente a $25 \times 25$ $\mathrm{km}\left(625 \mathrm{~km}^{2}\right)$; sin embargo, debido a su forma irregular, algunos cuadrantes no tuvieron la misma área. Esta cuadrícula se utilizó para construir una matriz básica de datos en la que se registró presencia (codificados con 1) y ausencia (codificados con 0) de cada especie en cada cuadrante. Los datos se procesaron en el programa EstimateS 8.2, analizando la matriz con el estimador Chao 2 corregido y 100 aleatorizaciones sin reemplazo, para minimizar el efecto del orden de las muestras.

Para examinar la afinidad entre la avifauna, y con el objetivo de evitar sesgos en los análisis por ausencia de datos puntuales (Rojas-Soto et al., 2003), se elaboró una segunda matriz de presencia/ausencia de las especies por cada cuadrante de la cuadrícula, utilizando como datos de presencia los mapas de distribución potencial construidos mediante modelado de nicho ecológico (proyecto CONABIO CE-015; Navarro y Peterson, 2007). Se aplicó a la matriz el índice de distancia de Bray-Curtis (Bray y Curtis, 1957) como medida de disimilitud. Este índice no da valor a las ausencias compartidas entre cada unidad clasificatoria, y es el adecuado para este tipo de estudios en los que se trabaja con datos de ausencias no confirmadas (De Klerk et al., 2002). Los valores obtenidos con este índice varían entre 0 y 1 , donde 0 representa la mínima disimilitud y 1 la máxima disimilitud.

Con los datos resultantes se obtuvo la matriz de disimilitud y se aplicó el método de ligamiento no ponderado mediante centroides (UPGMC, por sus siglas en inglés; Crisci y López, 1983). Con la matriz resultante se construyó el fenograma que muestra la similitud de la avifauna entre cada cuadrante. Como medida de la bondad de ajuste de los datos, logrado a partir del análisis de agrupamiento, se obtuvo el coeficiente de correlación cofenético. Para los análisis y gráficos se utilizó el programa NTSYS 2.02c (Rohlf, 1997). 


\section{Resultados}

El total de registros fue de 10983 , distribuidos en 857 localidades dentro de la península de Yucatán. En cuanto a la riqueza avifaunística, se obtuvo un total de 436 especies pertenecientes a 67 familias y 21 órdenes. Se registraron 22 especies con algún tipo de endemismo (4.6\% del total de especies registradas); 5 son endémicas de México y 17 cuasiendémicas. Las endémicas se distribuyen en la parte norte de los estados de Yucatán y Quintana Roo, aunque también se localizaron en la isla Cozumel. Las especies cuasiendémicas se distribuyen en el resto de la península de Yucatán, incluyendo las porciones norte de Belice y Guatemala. En relación con la estacionalidad, de las 436 especies totales registradas en la región, sólo 298 son residentes, lo que equivale al $68.3 \%$ del total de especies. Aunque la mayoría de las especies de aves de la península son residentes, al menos el 34.4\% (150 especies) son migratorias invernantes, siendo éste el componente migratorio más importante, junto con las aves transitorias, las cuales constituyen el 28.6\% (125 especies). El componente estacional de menor proporción lo conforman las aves migratorias de verano y las ocasionales-accidentales cuyos porcentajes respecto al total de las especies son del $2.5 \%$ (11 especies) y el $4.5 \%$ (20 especies), respectivamente. Algunas especies se encuentran incluidas en más de una categoría de estacionalidad, principalmente las migratorias de invierno con poblaciones residentes; por ejemplo, el flamenco Phoenicopterus ruber.

A partir de la aplicación del estimador de riqueza Chao2, utilizando como muestras los cuadrantes de $0.25^{\circ}$ $\times 0.25^{\circ}$ (en total 222), la riqueza estimada para la península de Yucatán fue de $441.07 \pm 7.37$ (DE) especies (Fig. 2). Si se toma en cuenta este resultado y el número de especies en la base de datos (436), lo que falta por registrar oscila en 11.44 especies (aproximadamente 2.6\%).

En el fenograma obtenido, usando el índice de distancia de Bray-Curtis y el algoritmo de agrupamiento UPGMC sobre datos de presencia y ausencia de la avifauna (Fig. 3), en los cuadrantes construidos (Fig. 4a) se obtuvo un coeficiente de correlación de 0.78699 , y se observan las similitudes faunísticas entre los 222 cuadrantes. Se reconocieron 4 agrupaciones principales. La primera se forma a un valor de distancia Bray-Curtis (BC) de 0.90, e incluye los cuadrantes que abarcan el área costera del extremo norte de Yucatán y Quintana Roo, y un cuadrante constituido por el área de la laguna de Bacalar; esta agrupación se denominó "zona norte"(en negro, Fig. 3); la segunda agrupación $(\mathrm{BC}=0.73)$, donde se identificaron, a su vez, 4 subnúcleos con cuadrantes correspondientes a las áreas más secas de la península, el bosque tropical caducifolio y el bosque tropical subcaducifolio; las áreas húmedas de
Sian Ka'an, se denominaron "zona centro" (en gris claro, Fig. 3); el tercer núcleo $(\mathrm{BC}=0.73)$, "zona sur" (en gris oscuro, Fig. 3), está conformado por los cuadrantes que ocupan el bosque tropical perennifolio; el área más húmeda de la península, con 3 subnúcleos: área de manglares al oeste, área de la Reserva de la Biosfera de Calakmul y el área que la circunda; la cuarta asociación sólo incluye isla Cozumel (cuadrante 222), que es el área con las mayores similitudes del fenograma $(\mathrm{BC}=0.05)$.

A partir de los resultados obtenidos, se elaboró el mapa de la figura $4 \mathrm{~b}$ en el que se observa la división principal de la península de Yucatán en 4 zonas: norte (negro), centro (gris claro), sur, y la isla Cozumel (gris oscuro). La zona con el mayor número de especies es la sur, con 401 especies $(92 \%$ del total); en la zona central se reconocen 305 especies, y 290 en isla Cozumel; finalmente, en la zona norte (negro) se registraron 150 especies. Estos cuadrantes son los mismos donde se encontró menor número de especies registradas, y por lo tanto, el menor número de especies presentes. En general, se registraron más de 100 especies en la mayoría de los cuadrantes.

\section{Discusión}

Con la construcción de la base de datos, se encontró que en la península de Yucatán se distribuyen al menos 436 especies de aves para las cuales existen registros confirmados por ejemplares. Esta cantidad difiere de la de otros trabajos en los que se reconocen mayor o menor número de especies. Por ejemplo, Paynter (1955a) registró 429 especies; Hartig (1979), 491 y MacKinnon (1992), 509. Todos estos trabajos fueron realizados mediante trabajo en campo y también utilizando listas publicadas; ninguno de ellos utilizó bases de datos recopiladas a partir de varias colecciones científicas y mucho menos un estimador de riqueza estadístico; además, según cada autor y año de publicación, se reconocen especies diferentes. Gran parte de la riqueza encontrada en la península de Yucatán se debe a la presencia de un importante número de especies de aves migratorias de invierno que utilizan esta porción continental como zona de residencia durante el invierno, o como área de paso y descanso en el seguimiento de sus rutas migratorias (Lynch, 1989; Greenberg, 1992). La importancia del componente migratorio, principalmente de invierno y transitorio, apreciada en trabajos anteriores, representa alrededor del 30\% de la avifauna registrada (López-Ornat et al., 1989; Lynch, 1989). Aunque este porcentaje varía ligeramente según las localidades y zonas estudiadas, se ajusta de manera coherente con el resultado de este trabajo, en el que la proporción es del $28.6 \%$.

De acuerdo con el trabajo de Lynch (1989), la distribución de especies invernantes y transitorias abarca toda 


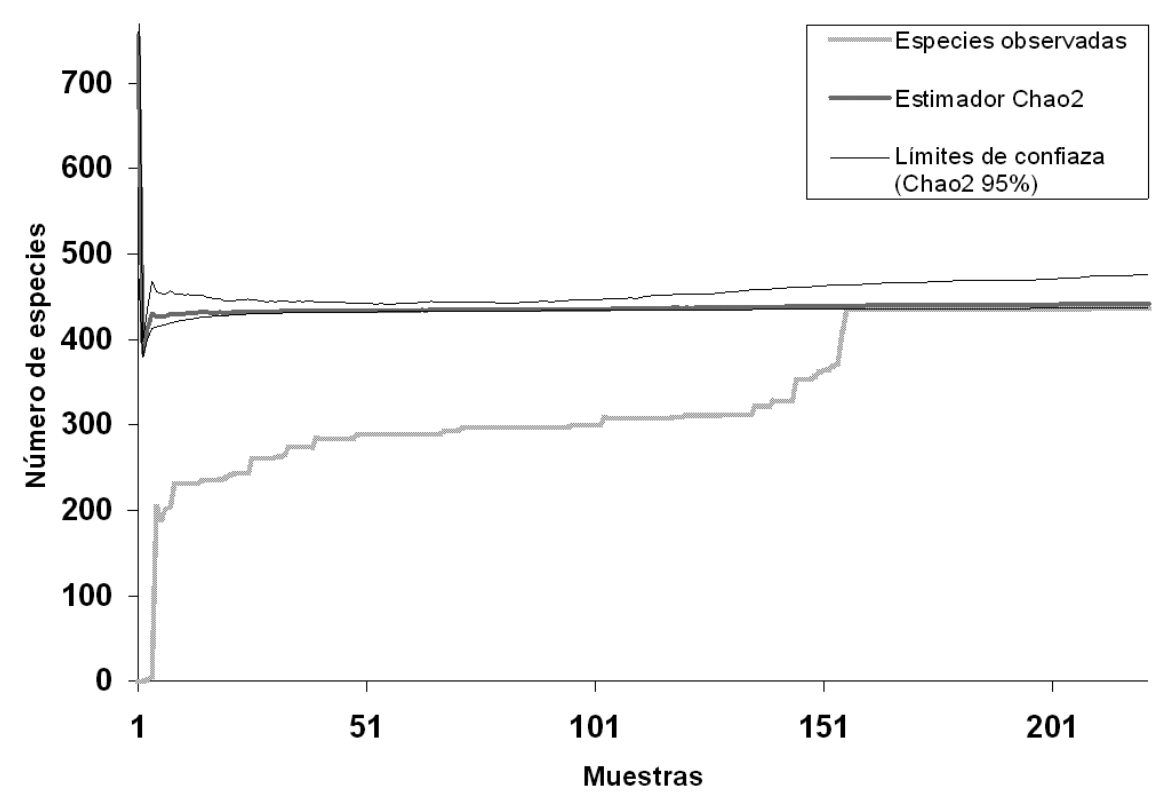

Figura 2. Estimación de la riqueza de especies en la península de Yucatán mediante el uso del estimador Chao2.

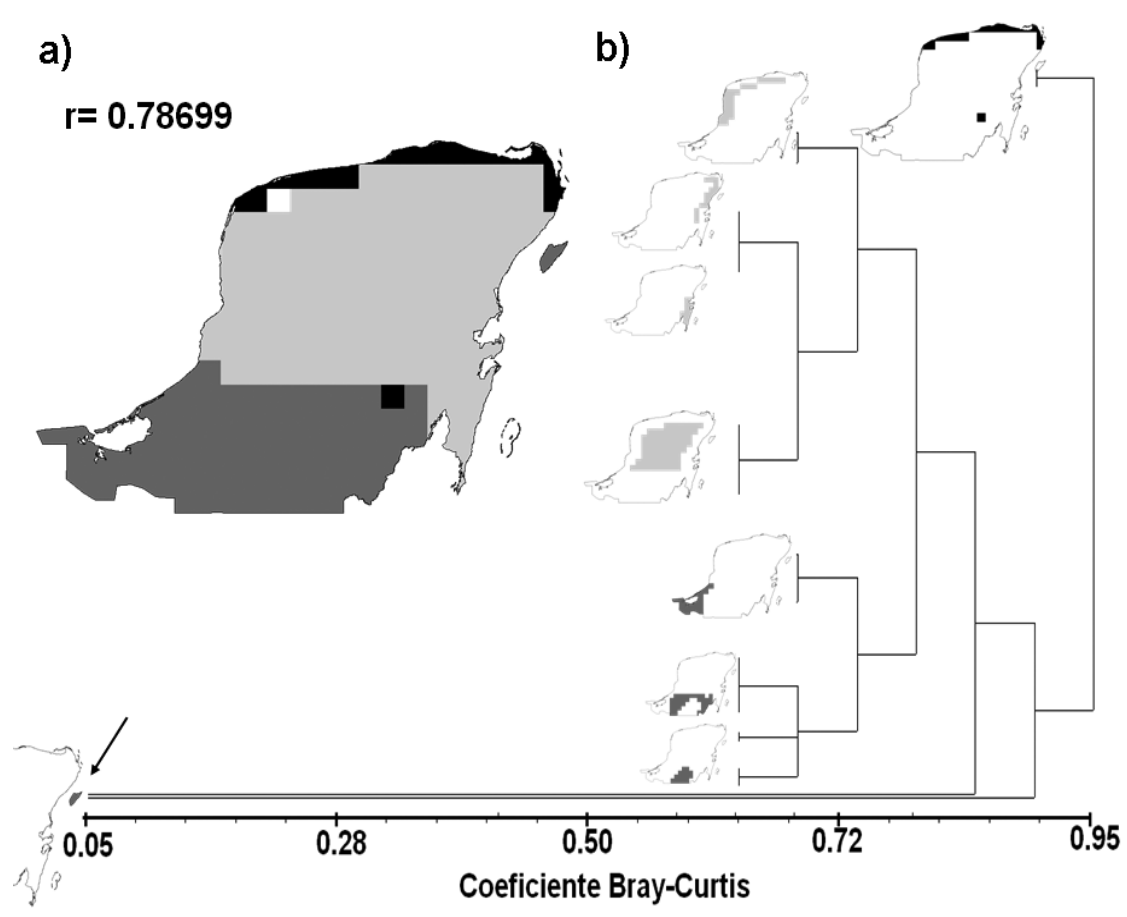

Figura 3. a), zonas definidas a partir del análisis de similitud; b), resumen de dendograma de relaciones entre cuadrantes, definido por la riqueza de especies de aves en la península de Yucatán mediante el uso del índice de distancia de Bray-Curtis y el algoritmo de agrupamiento UPGMC. En cada agrupación se muestra el área geográfica que conforma. r= coeficiente de correlación cofenético. 


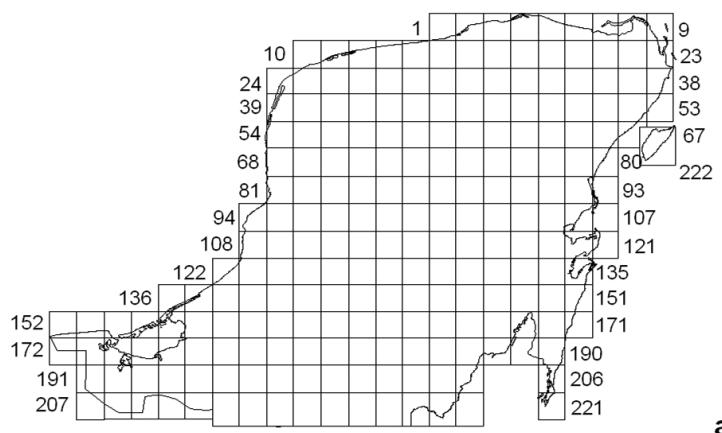

a)

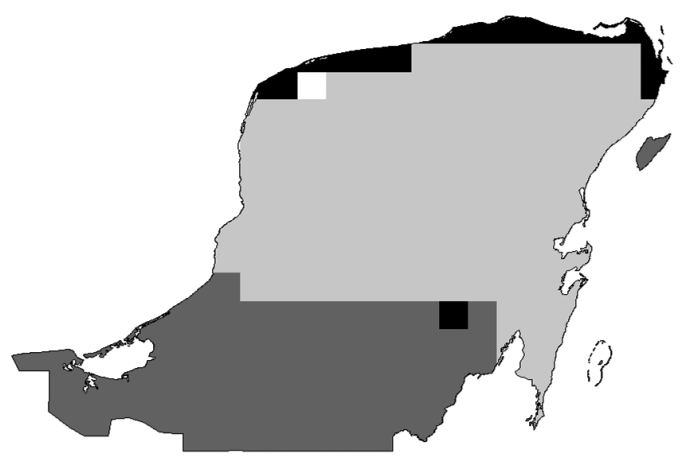

b)

Figura 4. a), cuadrantes utilizados para el análisis de similitud. El número de cuadrante se indica en los extremos de la cuadrícula; b), zonas definidas a partir del análisis de similitud.

el área de la península. Para Deppe y Rotenberry (2005), esta distribución se ve afectada por factores que incluyen la distribución de la vegetación, abundancia de alimento y competencia; cada uno influido por la edad, sexo y condición energética. También se ha notado que la mayor parte de las aves invernantes son de hábitos neárticos insectívoros (Greenberg, 1992), seguidas por los nectarívoros y frugívoros, omnívoros y granívoros (Levey y Stiles, 1992; Deppe y Rotenberry, 2005); aunque también se han detectado los cambios de hábitos alimenticios; por ejemplo, de una dieta de insectos a otra frugívora (Levey y Stiles, 1992; Greenberg et al., 1993), situación que se explica por la disminución en las poblaciones de insectos durante el invierno (Levey y Stiles, 1992).

La península no es especialmente rica en endemismos de aves en el nivel de especie. Arita y Vázquez-Domínguez (2003) indican, por ejemplo, que existen de 20-26 especies endémicas de anfibios y reptiles. En este trabajo sólo se encontraron 5 especies de aves endémicas de México. Este resultado concuerda con Escalante et al. (1998) quienes informan que por lo menos hay 5 especies endémicas de la península. El uso de taxonomías alternativas (e.g., Navarro-Sigüenza y Peterson, 2004; Peterson y Navarro-
Sigüenza, 2009) permite visualizar de manera diferente el endemismo en la península. Con otro enfoque, es posible reconocer 15 especies endémicas más para la península de Yucatán y en Cozumel, la riqueza de endémicas se incrementa a 6 especies. En total, el endemismo aumentaría a 37 especies, lo que haría de esta zona un sitio relevante de endemismo y diferenciación evolutiva.

El endemismo se ubica geográficamente de la misma manera que otros grupos de organismos (Arita y VázquezDomínguez, 2003; Espadas-Manrique et al., 2003). Las especies endémicas se distribuyen concentradas en una franja al norte de la península y en isla Cozumel. La única especie de ave endémica de México, que no lo es también de la península de Yucatán, es el colibrí Doricha eliza, pues esta especie posee 2 poblaciones disyuntas; la de mayor número es la que se encuentra en el área de estudio y la más pequeña, en una área reducida del centro de Veracruz (Ortiz-Pulido et al., 2002).

Esta distribución en la parte norte de la península puede sugerir que la diferenciación y establecimiento de estas formas fue reciente. El encontrarse en la parte más joven y relativamente estable de la península, en lo que a situación ambiental se refiere, promovió la aparición y expansión del bosque tropical seco más joven del país (Becerra, 2005). La diferencia de condiciones actuales junto con las oscilaciones climáticas del Pleistoceno (hace 36000 años) que se sufren más marcadamente al sur (Metcalfe et al., 2000), pudieron permitir la diferenciación de especies asociadas a ellas y, debido a la forma de la península, esta zona quedó relativamente aislada de la parte sur por el gradiente de humedad oeste-este, conformando una isla climática (Estrada-Loera, 1991).

En el caso de Cozumel -cuya historia geológica aún no se conoce bien-, al ser una isla cercana al continente, de alguna manera mantiene aisladas las poblaciones de ciertas especies. En la isla existen condiciones climáticas un tanto diferentes a las de las partes continentales más cercanas que incluyen mayor precipitación y humedad. Por otro lado, la avifauna de Cozumel se compone principalmente de elementos con afinidad antillana (incluyendo Centroamérica) y norteamericana cuya presencia se explica principalmente por eventos de dispersión (Paynter, 1955a; Vázquez-Miranda et al., 2007).

Las especies cuasiendémicas se distribuyen en toda la península, lo que indica que en su totalidad (incluyendo Guatemala y Belice) esta área ha tenido una historia biológica y geológica tan particular que ha permitido la diferenciación de especies propias de la provincia biogeográfica. Durante el Oligoceno, al formarse las cadenas montañosas de Chiapas y Centroamérica, las faunas del este y el oeste de México se separaron, permitiendo la diferenciación de sus elementos, como ya se sugirió para 
reptiles (Savage, 1982). Se ha notado que especies cuasiendémicas, como Uropsila leucogastra y Cyanocorax yucatanicus, representan especies hermanas de otras que se distribuyen al oeste de México (Navarro, 1998).

En general, el hecho de que existan tan pocas formas endémicas en la provincia puede deberse a las oscilaciones climáticas pleistocénicas que no permitieron estabilidad climática al sur de la península; además, esta área forma parte del puente centroamericano, por lo que se ha establecido un gran número de especies provenientes del sur y en menor medida del norte (Vuilleumier, 1985). Es decir, esta porción de tierra no ha estado lo suficientemente aislada como para permitir la diferenciación de muchas especies. Aún así, investigadores como Savage (1982) y Peterson et al. (1998), reconocen la península de Yucatán como una de las áreas de mayor endemismo de Centroamérica.

Dado que la base de datos no incluye la totalidad de las especies que en la realidad se distribuyen en toda la península, la lista de especies construida a partir de ella es incompleta. Por esta razón se recomienda el uso de estimadores de riqueza útiles para calificar qué tan completa es una lista (Escalante et al., 2002). En este trabajo se estimó que la riqueza de especies de la península oscila alrededor de las 441 especies, lo que indica que faltarían por registrar al menos 5 especies en las colecciones. Tomando en cuenta la distribución de las aves de Norteamérica presentada en Howell y Webb (1995) es posible hallar al menos 43 especies cuyas probables áreas de distribución abarcan o alcanzan la península de Yucatán. Sin embargo, no se han incluido en este trabajo porque de ninguna se encontraron registros respaldados con ejemplares; además, los mapas de Howell y Webb están trazados a mano utilizando la experiencia personal y conocimiento de las especies por parte de los autores. De haberse podido añadir, la riqueza de especies de aves hubiera aumentado de 436 a 479, superando el número de especies reconocidas en algunos trabajos antes mencionados, y acercándose al límite de confianza superior calculado para los resultados del estimador Chao2 (Fig. 2). Estos resultados podrían indicar que el muestreo llevado a cabo hasta ahora ha permitido registrar una gran proporción de las especies en la península y que son pocas las no registradas, aunque siguen apareciendo nuevos registros (e.g., Ibañez-Henández, 2000; Howell et al., 2001; Collins, 2003; MacKinnon et al., 2003; MacAndrews et al., 2006; Correa Sandoval et al., 2006; Chablé-Santos et al., 2011).

La península de Yucatán como provincia biótica. A través de los años, las particulares características bióticas y físicas de la península de Yucatán han sustentado su definición como una provincia florística, faunística, geológica, fisiográfica y biogeográfica (Barrera, 1962; Rzedowski, 1978; Ferrusquía-Villafranca, 1998; Espadas-Manrique et al.,
2003; Morrone y Márquez, 2003; Ramírez-Barahona et al., 2009). En cuanto a la biota, Barrera (1962) nota que esta consideración se había hecho sin realizar estudios cuantitativos. Por suerte, en los últimos años se han realizado trabajos que analizan cuantitativamente la flora y fauna de la región, obteniendo resultados más finos y documentados para varios taxa, para definirla como una provincia biótica (Barrera, 1962; Casas-Andreu y Reyna-Trujillo, 1990; Ramírez-Pulido y Castro-Campillo, 1990; IbarraManríquez et al., 2002; Ramírez-Barahona et al., 2009).

Aunque estos estudios confirman la identidad de la península de Yucatán como provincia biogeográfica, entre ellos difieren en mayor o menor medida tanto en el número como en la extensión de subáreas (distritos) distinguidos en ella. En este trabajo, el análisis de similitud por cuadrantes mostró que presenta 4 zonas distintivas en su avifauna. La primera, al norte, está definida por una mayor aridez, menor precipitación, suelos calcáreos de origen reciente con poca capacidad de retener humedad, bosque tropical seco y áreas de dunas costeras. La segunda área incluye la zona de bosque tropical caducifolio y bosque tropical subcaducifolio que son menos secas, tienen menos elementos bióticos acuáticos y comparten más elementos con los del bosque tropical perennifolio. La zona sur, tercera área, se distingue por presentar mayor humedad y precipitación, suelos de rendzina capaces de retener humedad y bosque tropical perennifolio (Rzedowski, 1978; García, 1988; Duch, 1991). Finalmente, la cuarta área o isla Cozumel es bosque tropical subcaducifolio pero, como se mencionó, es una isla, así que además de compartir elementos parecidos a los de la segunda área, tiene elementos antillanos y uno pequeño, endémico (Vázquez-Miranda et al., 2007).

Los altos valores de disimilitud presentados en el fenograma de la figura 3, podrían ser atribuidos a que la fauna de la península presenta grandes diferencias entre el norte y sur, sino más bien al muestreo tan heterogéneo e incluso escaso, así como al tamaño de la cuadrícula elegida que puede complicar las interpretaciones. El uso de los modelos de nicho ecológico ayuda a amortiguar este efecto con hipótesis de distribución continua que minimiza el sesgo que el uso de distribuciones puntuales puede provocar. (Rojas-Soto et al., 2003). Por lo tanto, los altos valores de disimilitud pueden estar reflejando realmente avifaunas ecológicamente distinguibles y de afinidades diferentes en la península de Yucatán. En cuanto a la baja cantidad de registros, no sólo es cierto para el caso de las aves. Escalante et al. (2002) encuentran que con mamíferos terrestres el número de registros por $\mathrm{km}^{2}$ en la península de Yucatán refleja un bajo esfuerzo de muestreo; mientras que Espadas-Manrique et al. (2003), con datos de árboles verifican la necesidad de mayor exploración en varias áreas y que los esfuerzos de colecta aún son insuficientes por sí 
mismos para identificar patrones históricos como las áreas de endemismo.

En este trabajo se observa que la zona sur alberga 401 especies, la norte y centro 150 y 305 , respectivamente, lo que indica que la riqueza de especies en la península de Yucatán disminuye de la base a la punta (i.e., de sur a norte), situación esperada por el llamado "efecto peninsular", que se refiere a la disminución de la diversidad y riqueza de especies en función de la distancia a la base de la península (Simpson, 1964) y se ha detectado en diferentes grupos de la provincia (Arita y Vázquez-Domínguez, 2003). Éste se debe a que el tipo de hábitat cambia de la base a la punta, haciéndose menos adecuado para cierto tipo de especies y más para otras (Means y Simberloff, 1987). Las afinidades de la avifauna dentro de la península no están determinadas por la presencia de barreras geográficas sino climáticas, particularmente por la diferenciación climática del norte con el sur y la vegetación característica de cada zona (Barrera, 1962; Ibarra-Manríquez et al., 2002; Ramírez-Barahona et al., 2009).

Finalmente, una comparación entre los resultados obtenidos en este trabajo y los realizados con otros grupos bióticos en la península de Yucatán muestran que las mayores diferencias se observan con el trabajo de Barrera (1962; Fig. 5c). En el primero se reconocen 3 distritos de extensiones muy diferentes a las del presente trabajo. Para realizar su estudio, Barrera (1962) utilizó los datos de distribución de peces, anfibios, reptiles y mamíferos no voladores conocidos hasta entonces, y los analizó con el coeficiente de similitud de Simpson; encontró que el distrito del norte se extiende más al sur que en el resto de los trabajos, y el distrito del sur ocupa el resto de la península, incluyendo Guatemala y Belice. De manera similar a este estudio, Barrera reconoce la isla de Cozumel como un tercer distrito.

Los resultados obtenidos se parecen más a los que se han presentado para árboles (Ibarra-Manríquez et al., 2002; Fig. 5d), helechos y licopodios (Ramírez-Barahona et al., 2009; Fig. 5b), ya que en ellos se reconocen sólo las áreas diferenciadas al noroeste y al sureste, como en el presente trabajo. Sin embargo, Ramírez-Barahona et al. (2009), basándose en un análisis de trazos y de parsimonia de endemismos, definen 4 subdistritos en la zona sureste (El Petén): Campeche, Calakmul, Quintana Roo y Sian Ka'an; mientras que, en la zona noroeste (Yucatán seco) definen 2: central y noroeste.

El trabajo más parecido es el elaborado por RamírezBarahona et al. (2009), que podría deberse a que utilizaron datos de distribución potencial de plantas. Aunque considerando que no dividen el área en cuadrantes ni utilizan
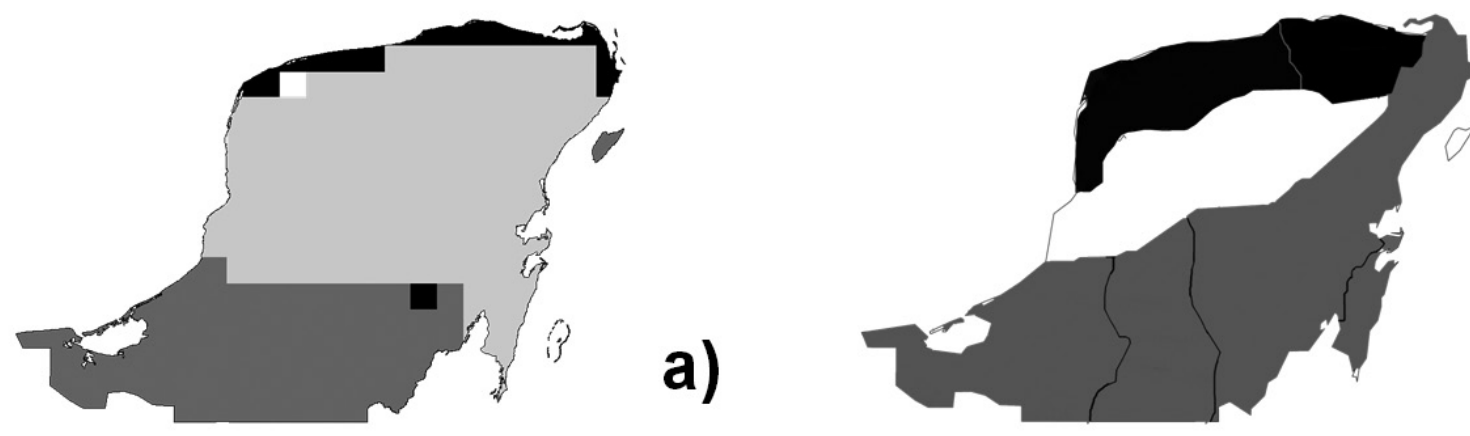

b)
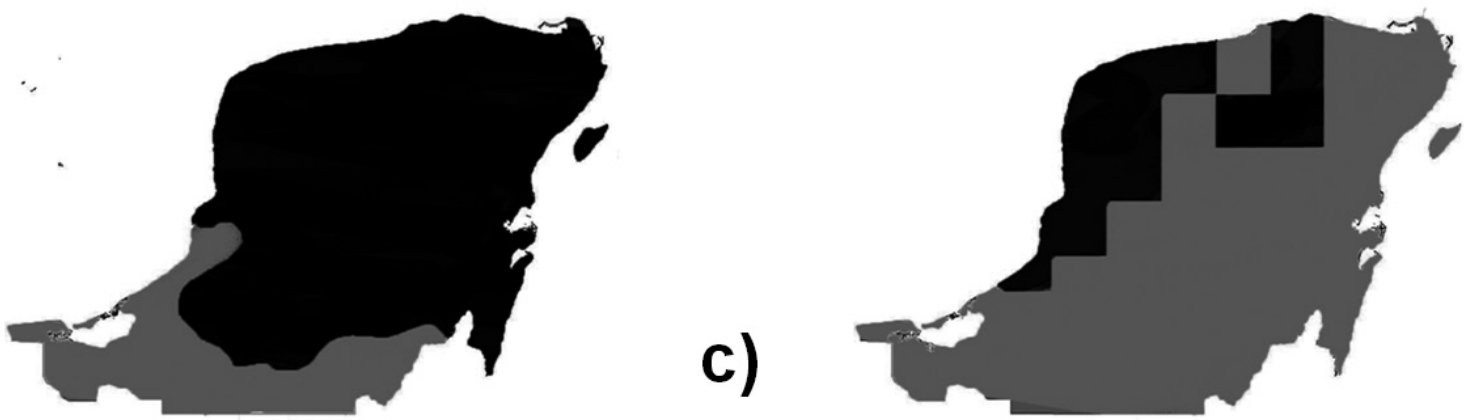

d)

Figura 5. Distintas representaciones de subdivisión de la provincia biótica de la península de Yucatán a partir de: a), aves (presente estudio); b), licopodios y helechos (Ramírez-Barahona et al., 2009); c), vertebrados (Barrera, 1962) y d), árboles (Ibarra-Manríquez et al., 2002). 
un análisis de similitud (los autores elaboran un análisis de trazos), el hecho de obtener distritos o subdivisiones tan similares a los de este trabajo corrobora la identidad de la avifauna de la península de Yucatán cuya distribución se relaciona geográficamente con la del bosque tropical caducifolio y perennifolio. Las diferencias entre los mapas presentados en las figuras $5 \mathrm{a}$ y b, se deberían entonces al método para analizar los datos y al sistema biológico con el que se trabajó en cada uno.

Aun a escalas espaciales mayores, la península de Yucatán presenta su propia identidad biótica con respecto a la avifauna; por ejemplo, Peterson et al. (1998) analizan la similitud entre la avifauna de Centroamérica mediante el método de ligamiento promedio no ponderado (UPGMA), encontrando una diferencia, aunque de diferente extensión, entre la avifauna de la zona norte y sur de la península. Es probable que no hayan encontrado más distritos debido a la escala del área de estudio y a la resolución de sus datos de distribución (presencia y ausencia determinada según las descripciones de AOU, 1983).

En conclusión, aunque varios trabajos difieren en su extensión, la mayoría sustenta la existencia de 2 áreas con faunas más características, una al norte y otra al sur. Las diferencias entre éstas no parece ser muy grande pues en general, la biota de la península de Yucatán es muy parecida, aunque es poco probable que alguna vez se logre una regionalización concordante con todos los grupos biológicos (Ibarra-Manríquez et al., 2002).

El análisis de los patrones geográficos y ecológicos de la distribución de las aves a partir de información contenida en las colecciones, es importante ante las necesidades y acciones de conservación. Sin esta información es imposible identificar las prioridades reales al respecto. Aunque es preciso aumentar la información contenida en las colecciones, los datos parecen ser suficientes para la comparación de patrones peninsulares de las aves con otros taxa.

\section{Agradecimientos}

A los curadores de las colecciones científicas de las siguientes instituciones: American Museum of Natural History, Academy of Natural Sciences of Philadelphia, Bell Museum (University of Minnesota), Natural History Museum (British Museum), Canadian Museum of Nature, Carnegie Museum of Natural History, Colección Nacional de Aves (Instituto de Biología, UNAM), Colección de Vertebrados Terrestres (Instituto Politécnico Nacional), Denver Museum of Natural History, Delaware Museum of Natural History, Fort Hays State College, Field Museum of Natural History, Leiden Natuurhistorische Museum, Louisiana State University Museum of Zoology, Museum of Comparative Zoology (Harvard University), Moore
Laboratory of Zoology, Museo de Historia Natural de París, Museum of Vertebrate Zoology (Berkeley Univ.), Museo de Zoología (Facultad de Ciencias, UNAM), Royal Ontario Museum, San Diego Natural History Museum, Southwestern College, University of Arizona, University of British Columbia, University of California Los Angeles, United States National Museum y Peabody Museum (Yale University), por brindarnos el acceso a sus datos. El apoyo financiero para la construcción de la base de datos del Atlas y la elaboración de mapas fue obtenido de CONABIO (A002, E018, CE 015), CONACYT, DGAPA-UNAM (IN 218598 y 214200), British Council México, National Science Foundation y la Comisión de Cooperación Ambiental para América del Norte. El apoyo para el desarrollo de este proyecto se obtuvo de PAPIIT (IN 208906) y SEMARNAT-CONACYT (C01-0265).

\section{Literatura citada}

AOU (American Ornithologists'Union). 1983. Checklist of North American birds. Washington, D.C. 877 p.

AOU (American Ornithologists' Union).1998. Check-list of North American birds, séptima edición. Washington D. C. $829 \mathrm{p}$.

AOU (American Ornithologists' Union). 2000. Forty-second supplement to the American Ornithologists' Union check-list of North American birds. Auk 117:847-858.

Arita, H. T. y E. Vázquez-Domínguez. 2003. Fauna y la conformación de la provincia biótica yucateca: biogeografía y macroecología. In Naturaleza y sociedad en el área maya. Pasado, presente y futuro, M. P. Colunga-García y A. LarquéSaavedra (eds.). Academia Mexicana de Ciencias y Centro de Investigación Científica de Yucatán, México. p. 69-80.

Banks, R. C., C. Cicero, J., L. Dunn, A. W. Kratter, P. C. Rasmussen, J. V. Remsen, J. D. Rising y D. F. Stotz. 2002, Forty-third supplement to the American Ornithologists' Union check-list of North American birds. Auk 119:897-906.

Banks, R. C., C. Cicero, J. L. Dunn, A. W. Kratter, P. C. Rasmussen, J. V. Remsen, J. D. Rising y D. F. Stotz. 2003. Forty-fourth supplement to the American Ornithologists' Union check-list of North American birds. Auk 120:923-931.

Banks, R. C., C. Cicero, J. L. Dunn, A. W. Kratter, P. C. Rasmussen, J. V. Remsen, J. D. Rising y D. F. Stotz. 2004. Forty-fifth supplement to the American Ornithologists' Union check-list of North American birds. Auk 121:985-995.

Banks, R. C., C. Cicero, J. L. Dunn, A. W. Kratter, P. C. Rasmussen, J. V. Remsen, J. D. Rising y D. F. Stotz. 2005. Forty-sixth supplement to the American Ornithologists' Union check-list of North American birds. Auk 122:1026-1031.

Banks, R. C., C. Cicero, J. L. Dunn, A. W. Kratter, P. C. Rasmussen, J. V. Remsen, J. D. Rising y D. F. Stotz. 2006. Forty-seventh supplement to the American Ornithologist' 
Union check-list of North American birds. Auk 123:926-936.

Banks, R. C., R. T. Chesser, C. Cicero, J. L. Dunn, A. W. Kratter, I. J. Lovette, P. C. Rasmussen, J. V. Remsen, J. D. Rising y D. F. Stotz. 2007. Forty-eighth supplement to the American Ornithologist' Union check-list of North American birds. Auk 124:1109-1115.

Banks, R. C., R. T. Chesser, C. Cicero, J. L. Dunn, A. W. Kratter, I. J. Lovette, P. C. Rasmussen, J. V. Remsen, J. D. Rising, D. F. Stotz y K. Winker. 2008. Forty-ninth supplement to the American Ornithologists' Union check-list of North American birds. Auk 125:758-768.

Barrera, A. 1962. La Península de Yucatán como provincia biótica. Revista de la Sociedad Mexicana de Historia Natural 23:71-105.

Becerra, J. X. 2005. Timing the origin and expansion of the Mexican tropical dry forest. Proceedings of the National Academy of Sciences 102:10919-10923.

Beltrán, E. 1958. Datos geográficos. In Los recursos naturales del sureste y su aprovechamiento, tomo II, E. Beltrán (ed.). Instituto Mexicano de Recursos Naturales Renovables, México, D. F. p. 3-11.

Boucard, A. 1883 On a collection of birds from Yucatan. Proceedings of the Zoological Societyt of London 1883:434462.

Bray, J. R. y J. T. Curtis. 1957. An ordination of the upland forest communities of southern Wisconsin. Ecological Monographs 27:325-349.

Buskirk, W. H. 1968. The arrival of trans-gulf migrants on the northern coast of Yucatan in fall. MS thesis, Louisiana State University, Baton Rouge. 287 p.

Casas-Andreu, G. y T. Reyna-Trujillo. 1990. Provincias herpetofaunísticas. In Herpetofauna (anfibios y reptiles). IV. 8.6. Atlas Nacional de México, vol. II. Escala 1:8 000000. Instituto de Geografía, UNAM, México, D. F.

Chable-Santos, J., C. Selem-Salas y S. Hernández-Betancourt. 2011. Nuevos registros de Columbina minuta, Pionus senilis y Basileuterus culicivorus en el estado de Yucatán, México. Cotinga 33:76-77.

Chesser, R. T., R. C. Banks, F. K. Barker, C. Cicero, J. L. Dunn, A. W. Kratter, I. J. Lovette, P. C. Ramussen, J. V. Remsen, J. D. Rising, D. F. Stotz y K. Winker. 2009. Fiftieth supplement to the American Ornithologists' Union check-list of the North American birds. Auk 126:705-714.

Cole, L. J., G. M. Allen y T. Barbour. 1906. Vertebrata from Yucatan. Bulletin of the Museum of Comparative Zoology Harvard University 50:159.

Collins, C. T. 2003. Notable records of Loggerhead Shrike Lanius ludovicianus and Yellow-headed Blackbird Xanthocephalus xanthocephalus for the Yucatán Peninsula, Mexico. Cotinga 20:99.

Colwell, R. K. 2009. EstimateS: Statistical estimation of species richness and shared species from samples. Version 8.2.
User's Guide and application. http://purl.oclc.org/estimates; última consulta: 20.IV.2011.

Correa-Sandoval, J., A. De Alba-Bocanegra, M. Tuz-Novelo, H. Bahena-Basave, E. May-Canche y S. I. Pérez-Cabrera. 2006. Xanthocephalus xanthocephalus nuevo registro para la parte continental de la península de Yucatán, México. Huitzil 7:1819.

Crisci, J. V. y M. F. López.1983. Introducción a la teoría y la práctica de la taxonomía numérica. Programa Regional de Desarrollo Científico y Tecnológico, Secretaría General de la OEA, Washington, D.C. 132 p.

DeGraaf, R. M. y J. H. Rappole. 1995. Neotropical migratory birds. Natural history, distribution and population change. Comstock, New York. 676 p.

De Klerk, H. M., T. M. Crowe, J. Fjeldsa y N. D. Burgess. 2002. Biogeographical patterns of endemic terrestrial afrotropical birds. Diversity and Distributions 8:147-162.

Deppe, J. L. y J. T. Rotenberry. 2005. Temporal patterns in fall migrant communities in Yucatan, Mexico. Condor 107:228243.

Duch, G. J. 1991. Fisiografía del estado de Yucatán. Su relación con la agricultura. Universidad Autónoma de Chapingo. México, D. F. 229 p.

Dwight, J. y L. Griscom. 1927. A revision of the geographical races of the Blue Grosbeak (Guiraca caerulea). American Museum novitates 257:6.

Escalante, P., A. G. Navarro y A. T. Peterson. 1998. Un análisis geográfico, ecológico e histórico de la diversidad de aves terrestres de México. In Diversidad biológica de México: orígenes y distribución, T. P. Ramamoorthy, R. Bye, A. Lot y J. Fa (eds.). Universidad Nacional Autónoma de México, Instituto de Biología, México. p. 279-304.

Escalante, T., D. Espinosa y J. J. Morrone. 2002. Patrones de distribución geográfica de los mamíferos terrestres de México. Acta Zoológica Mexicana (n. s.) 87:47-65.

Espadas-Manrique, C., R. Durán y J. Argáez. 2003. Phytogeographic analysis of taxa endemic to the Yucatan Peninsula using geographic information systems, the domain heuristic method and parsimony analysis of endemicity. Diversity and Distributions 9:313-330.

ESRI. 1999. ArcView GIS ver. 3.2. Environmental Systems Research.

Estrada-Loera, E. 1991. Phytogeographic relationships of the Yucatan Peninsula. Journal of Biogeography 18:687-697.

Ferrusquía-Villafranca, I. 1998. Geología de México. Una sinopsis. In Diversidad biológica de México: orígenes y distribución, T. P. Ramamoorthy, R. Bye, A. Lot y J. Fa (eds.). Instituto de Biología, UNAM, México, D. F. p. 3-108.

García, E. 1988. Atlas Nacional del Medio Físico. Instituto Nacional de Estadística, Geografía e Informática, Aguascalientes, Aguascalientaes.

García, J. y J. Correa-Sandoval. 1988. Listado de las aves en el 
área de la Reserva Ría Lagartos, Yucatán, México. Pronatura, Península de Yucatán/SEDUE, Yucatán/ CINVESTAV, Mérida, Yucatán.

García, J., J. Correa-Sandoval y S. Vigilante. 1989. Listado de las aves en el área de la Reserva Ría Celestún, Yucatán, México. Pronatura Península de Yucatán/ SEDUE Yucatán/ CINVESTAV, Mérida, Yucatán.

Goldman, E. A. 1951. Biological investigations in Mexico. Smithsonian Miscellaneous Collections 115:1-476.

Goldman, E. A. y R. T. Moore. 1946. Biotic provinces of Mexico. Journal of Mammalogy 26:347-360.

Gómez-Pompa, A. 2004. The role of scientists in a troubled world. BioScience 54:217-226.

Greenberg, R. 1992. Forest migrants in non-forest habitats on the Yucatán Peninsula. In Ecology and conservation of Neotropical migrant landbirds, J. M. Hagan III y D. W. Johnston (eds.). Smithsonian Institute Press, Washington, D.C. p. 273-286.

Greenberg, R., D. K. Niven, S. Hopp y C. Boone. 1993. Frugivory and coexistence in a resident and a migratory vireo on the Yucatan Peninsula. Condor 95:990-999.

Griscom, L. 1926. The ornithological results of the MasonSpinden expedition to Yucatan. Part I: Introduction: birds of the main land of eastern Yucatan. American Museum Novitates 235:1-19.

Ibáñez-Hernández, G. 2000. Nuevos registros de aves para la costa este del estado de Yucatán, México. Cotinga 14:52-56.

Hands, S. y B. Everitt. 1987. A Monte Carlo study of the recovery of cluster structure in binary data by hierarchical clustering techniques. Multivariate Behavioral Research 22:235-243.

Hardy, J. W., R. J. Raitt, J. Orejuela, T. Webber y B. Edinger. 1975. First observation of the Orange-breasted falcon in the Yucatan Peninsula of Mexico. Condor 77:512.

Hartig, H. M. 1979. Las aves de Yucatán. Fondo Editorial de Yucatán. Mérida. Cuaderno 4.

Hernández-Barrios, J. C. y A. García de Fuentes. 2002. Análisis del impacto de la ganadería bovina sobre el medio ambiente de la península de Yucatán, a través de un sistema de información geográfica. In México en su unidad territorial, tomo I, Instituto Nacional de Estadística Geografía e Informática (ed.). Aguascalientes, Aguascalientes. p. 263-276.

Howell, S. N. G. 1989. Additional information on the birds of the Campeche Bank, Mexico. Journal of Field Ornithology 60:504-309.

Howell, S y S. Webb. 1995. Bird guide of Mexico and Northern Central America. Oxford University Press. 851 p.

Ibarra-Manríquez, G., J. L. Villaseñor, R. Durán y J. Meave. 2002. Biogeographical analisys of the tree flora of the Yucatan Peninsula. Journal of Biogeography 29:17-29.

Jonson, R. A. y P. S. Ward. 2002. Biogeography and endemism of ants (Hymenoptera: Formicidae) in Baja California, Mexico: a first overview. Journal of Biogeography 29:1009-1026.
Kluza, D. A. 1998. First record of Shiny Cowbird (Molothrus bonariensis) in Yucatán, Mexico. Wilson Bulletin 110:429430.

Levey, D. J. y F. G. Stiles. 1992. Evolutionary precursors of long-distance migration: resource availability and movement patterns in neotropical landbirds. American Naturalist 140:447-476.

López-Ornat, A. y C. Ramo. 1992. Colonial waterbird populations in the Sian Ka'an biosphere reserve (Quintana Roo, México). Wilson Bulletin 104:501-515.

López-Ornat, A., J. F. Lynch y B. MacKinnon de Montes. 1989. New and noteworthy records of birds from the eastern Yucatan Peninsula. The Wilson Bulletin 101:390-490.

Lynch, J. F. 1989. Distribution of overwintering Neartic migrants in the Yucatan Peninsula, I: General patterns of ocurrence. Condor 91:515-544.

Lynch, J. F. 1992. Distribution of overwintering Neartic migrants in the Yucatan Peninsula, II: Use of native and human-modified vegetation. In Ecology and conservation of Neotropical migrant landbirds, J. M. Hagan III and D. W. Johnston (eds.). Smithsonian Institute Press. p. 178-196.

Madariaga, C., M. L. y M. C. Yerena. 1981. Field checklist of the birds of the Yucatan Peninsula and its protected areas/ Lista de registros de campo de las aves de la península de Yucatán y sus áreas protegidas. In Memorias del 1er Simposio Internacional Familia Cracidae. Facultad de Medicina Veterinaria y Zootecnia, UNAM. Cocoyoc, Morelos. p. 62-71.

MacKinnon, H. 1989. 100 common birds of the Yucatán Peninsula. Amigos de Sian Ka'an, Cancún, Quintana Roo. $220 \mathrm{p}$.

MacKinnon, H. 1992. Field checklist of the birds of the Yucatan Preninsula and its protected areas/Listado para registros de campo de las aves de Yucatán y sus áreas protegidas. Amigos de Sian Ka'an, Cancún, Quintana Roo. 32 p.

MacKinnon, H. 1993. Ornitología de la península de Yucatán. Bibliografía anotada, primera edición. Amigos de Sian Ka’an, Cancún, Quintana Roo, México. 76 p.

MacKinnon, H., J. Laesser, J. Rotenberg y L. I. Tellez. 2003. Eremophila alpestris (Alaudidae): a new bird species and family for the Yucatan Peninsula, México. Huitzil 4:1-2.

McAndrews, A. E., J. E. Montejo-Díaz y M. Tabasco-Contreras. 2006. First confirmed record of the short-eared owl (Asio flammeus) for the state of Yucatan, Mexico. Huitzil 7:32-34.

Means, D. B. y D. Simberloff. 1987. The peninsula effect: habitat-correlated species decline in Florida's herpetofauna. Journal of Biogeography 14:551-568.

Metcalfe, S. E., S. L. O'Hara, M. Caballero y S. J. Davies. 2000. Records of late Pleistocene-Holocene climatic change in México- a review. Quaternary Science 19:699-721.

Morrone, J. J., D. Espinosa-Organista y J. Llorente-Bousquets. 2002. Mexican biogeographic provinces: preliminary 
scheme, general characterizations, and synonymies. Acta Zoológica Mexicana (n. s.) 85:83-108.

Morrone, J. J. y J. Márquez. 2003. Aproximación a un atlas biogeográfico mexicano: componentes bióticos principales y provincias biogeográficas. In Una perspectiva latinoamericana de la biogeografía, J. J. Morrone y J. Llorente Bousquets. CONABIO/ Las Prensas de Ciencias, UNAM, México, D. F. p. 217-220.

Myers, N. 1993. Tropical forests: the main deforestation fronts. Environmental Conservation 20:9-16.

Navarro, S. y H. Benítez. 1993. Patrones de riqueza y endemismo de las aves. Ciencias (Número especial) 7:45-53.

Navarro, A. G. 1998. Distribución geográfica y ecológica de la avifauna del estado de Guerrero, México. Tesis, Doctorado Facultad de Ciencias, Universidad Nacional Autónoma de México, México, D. F. 182 p.

Navarro-Sigüenza, A. G. y A. T. Peterson. 2004. An alternative species taxonomy of the birds of México. Biota Neotropica 4:1-32.

Navarro, A. G., A. T. Peterson y A. Gordillo-Martínez. 2003. Museums working together: the atlas of the birds of Mexico. In Why museums matter: avian archives in an age of extinction, N. Collar, C. Fisher y C. Feare (eds.). Bulletin British Ornithologists' Club Suplement 123A:207-225.

Navarro, A. G. y A. T. Peterson. 2007. Mapas de las aves de México basados en WWW. CONABIO (CE-015) http:// www.conabio.gob.mx; última consulta: 11.IV.2011.

Ortiz-Pulido, R., A. T. Peterson, M. B. Robbins, R. Díaz, A. G. Navarro-Sigüenza y G. Escalona-Segura. 2002. The mexican sheartail (Doricha eliza): morphology, behavior, distribution, and endangered status. Wilson Bulletin 114:153-160.

Parkes, K. C. 1997. The northern Cardinals of the Caribbean slope of Mexico, with the description of an additional subspecies from Yucatan. In The era of Alan R Phillips: a festschrift, R. W Dickerman (comp.). Horizon Communications, Albuquerque, New Mexico. p. 129-138.

Paynter, R. A. 1953. Autumnal migrants on the Campeche Bank. Auk 70:338-349.

Paynter, R. A. 1955a. The ornitogeography of the Yucatan Peninsula. Peabody Museum of Natural History Bulletin 9:1347.

Paynter, R. A. 1955b. Additions to the ornithogeography of the Yucatan Peninsula. Postilla 22:14.

Peters, J. L. 1913. List of the birds collected in the territory of Quintana Roo, Mexico, in the winter and spring of 1912. Auk 30:367-380.

Peterson, A. T., G. Escalona-Segura y J. A. Griffith. 1998. Distribution and conservation of the birds of northern Central America. Wilson Bulletin 110:534-543.

Peterson, A. T. y A. G. Navarro-Sigüenza. 2009. Constructing check-lists and avifauna-wide reviews: Mexican bird taxonomy revisited. Auk 126:915-921.
Ramírez-Barahona, S., A. Torres-Miranda, M. Palacios-Ríos e I. Luna-Vega. 2009. Historical biogeography of the Yucatan Peninsula, Mexico: a perspective from ferns (Monilophyta) and lycopods (Lycophyta). Biological Journal of the Linnean Society 98:775-786.

Ramírez-Pulido, J. y A. Castro-Campillo, 1990. Regiones y provincias mastogeográficas, Regionalización mastofaunística, IV. 8.8, escala 1:4 000 000. Atlas nacional de México, vol. II. Instituto de Geografía, UNAM, México, D. F.

Ridgway, R. 1881. On Amazilia yucatanesis (Cabot) and A. cerviniventris, Gould. Proceedings of the United States National Museum 4:25-26.

Ridgway, R. 1882. Descriptions of some new North American birds. Proceedings of the United States National Museum 5:343-346.

Ridgway, R. 1885a. Description of a new American kingfisher. Proceedings of the Biological Society of Washington 2:9596.

Ridgway, R. 1885b. Description of a new warbler from Yucatan. Proceedings of the United States National Museum 8:23.

Ridgway, R. 1885c. Description of some new species of birds from Cozumel Island, Yucatan. Proceedings of the Biological Society of Washington 3:21-24

Rodríguez-Yáñez, C. A., R. M.Villalón y A. G. Navarro 1994. Bibliografía de las aves de México (1825-1992). Facultad de Ciencias, Universidad Nacional Autónoma de México, México, D. F. 146 p.

Rogers, D. T. Jr., J. Garcia y A. Rogel. 1986. Additions to records of North American avifauna in Yucatan, Mexico. Wilson Bulletin 98:163-167.

Rohlf, J. 1997. Numerical taxonomy and multivariate analysis system (NTSYS). Exeter Software. New York.

Rojas-Soto, O. R., O. Alcántara-Ayala y A. G. Navarro. 2003. Regionalization of the avifauna of the Baja California Peninsula, Mexico: a parsimony analysis of endemicity and distributional modelling approach. Journal of Biogeography 30:449-461.

Rzedowski, J. 1978. Vegetación de México. Limusa, México, D. F. $432 \mathrm{p}$.

Salvin, O. 1888, 1889, 1890. A list of the birds of the islands of the coast of Yucatan and of the Bay of Honduras. Ibis:241265, 359-379, 84-95.

Salvin, O. y F. D. Godman. 1879-1904. Biologia Centrali Americana (Aves), vols. I, II, III. Taylor and Francis, London.

Sánchez, O. y R. Pérez-Hernández. 2005. Historia y tabla de equivalencias de las propuestas de subdivisiones biogeográficas de la región Neotropical. In Regionalización biogeográfica en Iberoamérica y tópicos afines. Primeras Jornadas Biogeográficas de la Red Iberoamericana de Biogeografía y Entomología Sistemática (RIBES XII.ICYTED), J. Llorente Bousquets y J. J. Morrone (eds.). 
CYTED/ UNAM/ CONABIO. México, D. F. p. 145-169.

Savage, J. M. 1982. The enigma of the Central American herpetofauna: dispersal or vicariance? Annals of the Missouri Botanical Garden 69:464-547.

SEMARNAT (Secretaría de Medio Ambiente y Recursos Naturales). 2002. Norma Oficial Mexicana NOM-059ECOL-2001. Protección ambiental-especies nativas de México flora y fauna silvestres-categorías de riesgo y especificaciones para su inclusión, exclusión o cambio-lista de especies en riesgo. Diario Oficial de la Federación, 6 de marzo.

Scott, P. E. y R. F. Martin. 1984. Avian consumers of Bursera, Ficus, and Erethia fruit in Yucatan. Biotropica 16:319-323.

Scott, P. R., D. D. Andrews y B. Mackinnon. 1985. Spotted Rail: first record from the Yucatan Peninsula. American Birds $5: 854$.

Simpson, G. G. 1964. Species density of North American recent mammals. Systematic Zoology 13:57-73.

Traylor, M. A. 1941. Birds from the Yucatan Peninsula. Publications of The Field Museum of Natural History, Zool.
Ser. 24:195-225.

Van Tyne, J. y M. B. Trautman. 1941. New birds from Yucatan. Occasional Papers University of Michigan. 439.

Van Tyne, J. y M. B. Trautman. 1945. Migration records from Yucatan. Wilson Bulletin 57:203-204.

Vázquez-Miranda, H., A. G. Navarro-Sigüenza y J. J. Morrone. 2007. Biogeographical patterns of the avifaunas of the Caribbean Basin Islands: a parsimony perspective. Cladistics 23:180-200.

Vuilleumier, F. 1985. Fossil and Recent Avifaunas and the Interamerican Interchange. In The great American biotic interchange, F. G. Stehli y S. D. Webb (eds.). Plenum, New York. p. 387-419.

Waide, R. B., J. T. Emlen y E. J. Tramer. 1980. Distribution of migrant birds in the Yucatan Peninsula: a survey. In Migrant birds in the Neotropics: Ecology, behavior, distribution and conservation, A. Keast y E. S. Morton (eds.). Smithsonian Institute Press, Washington, D.C. p. 165-171.

Waide, R. B. 1981. Interactions between resident and migrant birds in Campeche, Mexico. Tropical Ecology 22:134-154. 